\title{
LRRK2 Expression Is Deregulated in Fibroblasts and Neurons from Parkinson Patients with Mutations in PINK1
}

\author{
Garikoitz Azkona $^{1,2,3}$ - Rakel López de Maturana ${ }^{1}$ Patricia del Rio ${ }^{1}$ - Amaya Sousa ${ }^{1}$. \\ Nerea Vazquez ${ }^{1}$ • Amaia Zubiarrain ${ }^{1}$ • Daniel Jimenez-Blasco ${ }^{4,5}$ • Juan P. Bolaños ${ }^{4,5}$. \\ Blas Morales $^{6}$ • Georg Auburger ${ }^{7}$. José Matias Arbelo ${ }^{8}$ • Rosario Sánchez-Pernaute ${ }^{1,9}$
}

Received: 20 September 2016/Accepted: 16 November 2016/Published online: 14 December 2016

(C) The Author(s) 2016. This article is published with open access at Springerlink.com

\begin{abstract}
Mutations in PINK1 (PARK6), a serine/ threonine kinase involved in mitochondrial homeostasis, are associated with early onset Parkinson's disease. Fibroblasts from Parkinson's disease patients with compound heterozygous mutations in exon $7(\mathrm{c} .1488+1 \mathrm{G}>$ A; c.1252_1488del) showed no apparent signs of mitochondrial impairment. To elucidate changes primarily caused by lack of functional PINK1, we overexpressed wild-type $P I N K 1$, which induced a significant downregulation of LRRK2 (PARK8). Indeed, we found that LRRK2 protein basal levels were significantly higher in the mutant PINK1 fibroblasts. To examine the interaction between the two PARK genes in a disease-relevant cell context, we generated induced pluripotent stem cell (iPSC) lines from mutant, carrier and control fibroblasts by lentiviral-mediated re-programming. Efficiency of neural induction and dopamine
\end{abstract}

Garikoitz Azkona, Rakel López de Maturana, and Patricia del Rio contributed equally to this work.

Electronic supplementary material The online version of this article (doi:10.1007/s12035-016-0303-7) contains supplementary material, which is available to authorized users.

Rosario Sánchez-Pernaute

rpernaute@inbiomed.org; rossapernaute@gmail.com

1 Laboratory of Stem Cells and Neural Repair, Inbiomed, Paseo Mikeletegi, 81, 20009 San Sebastian, Spain

2 Animal Model Unit, Inbiomed, San Sebastian, Spain

3 Present address: Animal Research Facility, Scientific and Technological Centers, University of Barcelona, Barcelona, Spain

4 Institute of Functional Biology and Genomics (IBFG), University of Salamanca - CSIC, Salamanca, Spain differentiation using a floor-plate induction protocol was similar in all genotypes. As observed in fibroblasts, PINK1 mutant neurons showed increased LRRK2 expression both at the RNA and protein level and transient over-expression of wild-type PINK1 efficiently downregulated $L R R K 2$ levels. Additionally, we confirmed a dysregulation of $L R R K 2$ expression in fibroblasts from patients with a different homozygous mutation in PINK1 exon 4, c.926G > A (G309D). Thus, our results identify a novel role of PINK1 modulating the levels of LRRK2 in Parkinson's disease fibroblasts and neurons, suggest a convergent pathway for these PARK genes, and broaden the role of LRRK2 in the pathogenesis of Parkinson's disease.

Keywords Parkinson disease $\cdot$ iPSC $\cdot$ PINK1 $\cdot$ LRRK2
5 Institute of Biomedical Research of Salamanca (IBSAL), University Hospital of Salamanca, Salamanca, Spain

6 Department of Neurology, University Hospital San Cecilio, Granada, Spain

7 Experimental Neurology, Goethe University Medical School, Frankfurt am Main, Germany

8 Parkinson's and Movement Disorders Unit, Department of Neurology, Hospital Universitario Insular de Gran Canaria, Las Palmas de Gran Canaria, Spain

9 Present address: Andalusian Initiative for Advanced Therapies, Junta de Andalucia, Sevilla, Spain 


\section{Introduction}

Rare monogenic forms of Parkinson's disease (PD) have been correlated to specific gene mutations [1], providing the opportunity to identify novel pathogenic pathways or molecular mechanisms that may contribute to more frequent forms of the disease [2] and to examine interactions between PARK genes. Alternatively, early-onset genetic forms could represent a distinct entity at the molecular level.

PD-related mutations in PINK1 (PTEN-induced kinase 1, PARKO) compromise kinase function or protein stability [3]; thus, the phenotype is thought to result from a loss of function. PINK1 mitochondrial localization supports its involvement in the mitochondrial dysfunction extensively described in PD [4], and PINK1 protects against stress-induced mitochondrial dysfunction $[3,5]$. In addition, the PINK1/Parkin signaling pathway controls mitochondrial quality and mitophagy [6], even if it appears that this pathway is not modified by endogenous human PINK1 mutations [7, 8]. Likewise, PINK1 regulates mitochondrial fusion and fission dynamics [9], although the net effect of PINK1 mutations is currently a matter of controversy because both increased fusion and increased fission have been reported in different species [10].

LRRK2 (PARK8) is one of the genes most frequently mutated in PD. LRRK2 has been implicated in a very broad range of cellular pathways so the precise mechanisms leading to neuronal degeneration remain to be defined [11]. An effect of LRRK2 on mitochondrial function was described in Caenorhabditis elegans [12]. Interestingly, in this model organism, the absence of 1rk-1 rescues all phenotypic aspects of pink-1 loss-of-function mutants. Conversely, the hypersensitivity of lrk-1 mutant animals to stressors is reduced in a pink-1 mutant background, suggesting antagonistic effects of lrk-1 and pink-1 [13].

With the aim to define a PINK1-related phenotype for in vitro studies, we investigated expression pattern of genes involved in mitochondrial dynamics and PARK genes in fibroblasts and induced pluripotent stem cell (iPSC)-derived dopamine neurons from a PINK1-PD Spanish kindred [14]. This led us to unveil an upregulation of LRRK2 in PINK1 mutants and an interaction between these two PARK gene products in human cells which were ratified in a different pedigree.

\section{Methods}

\section{Human Samples}

Skin samples were obtained from subjects expressing mutated forms of PINK1 diagnosed at the Hospital Universitario Insular de Gran Canaria (La Palma de Gran Canaria, Spain) and from age-matched healthy individuals at the Hospital Donostia and Onkologikoa (San Sebastian, Spain). PD patients presented an early-onset, typical parkinsonian syndrome, characteristic of PINK1-associated PD [14]. Demographic data are provided in Table 1. Three samples from individuals from another Spanish family harboring the G309D (c.926G > A) mutation in exon 4 [3] were also analyzed to avoid possible confounding effects caused by consanguinity in the first family. Dermal fibroblasts were cultivated as described previously [15].
Table 1 Dermal fibroblast samples

\begin{tabular}{|c|c|c|}
\hline Human fibroblast samples & Age at biopsy & Pathology \\
\hline M-35 & $<55$ & None \\
\hline M-44 & $<55$ & None \\
\hline FH1103 & $<55$ & None \\
\hline FH0819 & $>55$ & None \\
\hline FH0821 & $>55$ & None \\
\hline 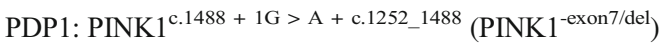 & $<55$ & PD \\
\hline PDP2: PINK1 $1^{\mathrm{c} .1488+1 \mathrm{G}>\mathrm{A}}\left(\mathrm{PINK} 1^{- \text {exon7 }}\right)$ & $<55$ & Asymptomatic \\
\hline 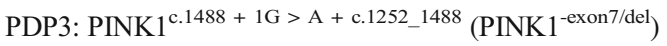 & $>55$ & $\mathrm{PD}$ \\
\hline PDP4: PINK1 $1^{\mathrm{c} .1488+1 \mathrm{G}>\mathrm{A}}\left(\mathrm{PINK} 1^{- \text {exon7 }}\right)$ & $<55$ & Asymptomatic \\
\hline PDP5: PINK1 G309D/G309D & $>55$ & $\mathrm{PD}$ \\
\hline PDP6: PINK1 G309D & $>55$ & Asymptomatic \\
\hline PDP7: PINK1 G309D/G309D & $<55$ & PD \\
\hline
\end{tabular}

The study included dermal fibroblasts from five control subjects (two males and three females) and seven subjects with mutations in PINK1 from two Spanish kindreds, four patients (two males and two females and three carriers (one male and two females) [14, 20]. Fibroblasts from the PDP1, PDP2, and PDP3 individuals were reprogrammed to obtain iPSCs lines and subsequently differentiated to dopamine neurons

$P D$ Parkinson disease 


\section{Protocol Approvals, Registrations, and Patient Consents}

The study was approved by the Ethical Committee on the Use of Human Subjects in Research in Euskadi, Spain. All subjects gave informed consent for the study using forms approved by the ethical committees on the Use of Human Subjects in Research at Hospital Universitario Insular de Gran Canaria, La Palmas de Gran Canaria; Hospital Donostia and Onkologikoa, San Sebastián; and Hospital San Cecilio, Granada, respectively. Generation of iPSC lines was approved by the Advisory Committee for Human Tissue and Cell Donation and Use, Instituto Carlos III, Ministry of Health, Spain.

\section{Genetic Analysis}

PINK1 variants were analyzed by conventional PCR using a primer pair designed to amplify a region expanding exons 6 and 8 [14]. Total RNA and cDNA were obtained as described previously [15] for quantitative RT-PCR. Primer sequences [14] are provided in Supplementary Table S1. Comparative analysis of gene expression levels $(\Delta \Delta \mathrm{Ct})$ was carried out using GAPDH as reference. Standard G-band karyotypes of the iPSC clones used in the study were performed at the Policlinica Gipuzkoa (San Sebastian, Spain).

\section{ATP Content}

Cellular ATP was measured using the Luminiscent ATP Detection kit (Abcam, Cambridge, UK). Cells were harvested, pelleted, and washed once in PBS. An aliquot was used for protein quantification. The rest was re-suspended in $50 \mu \mathrm{l}$ growth medium, and cells were lysed by adding $50 \mu \mathrm{l}$ of the detergent and mixing. After a 5-min incubation, $50 \mu \mathrm{l}$ of substrate was added and luminescence was quantified in a GloMax ${ }^{\circledR}$ luminometer (Promega, Madison, WI, USA). An ATP standard curve was prepared, and values were calculated in picomole per microgram of protein in the cell extract.

\section{Glycolytic Rate}

The rate of glycolysis was determined in fibroblasts, seeded in flasks, by the conversion of $\left[3-{ }^{3} \mathrm{H}\right]$ glucose into ${ }^{3} \mathrm{H}_{2} \mathrm{O}$, as described previously [16].

\section{Western Blotting}

Whole-cell lysates were prepared in RIPA buffer with a protease inhibitor cocktail (Roche, Mannheim, Germany). SDSPAGE and protein transfer and blotting were carried out according to standard procedures [15]. Primary and secondary antibodies are listed in Supplementary Table S2. Visualization of HRP-labeled proteins was performed using enzyme-linked chemifluorescence (ThermoFisher Scientific, Waltham, MA, USA) and quantified using ImageJ software. Data were normalized to control in order to compare different experiments.

\section{Immunofluorescence}

Cells plated onto glass coverslips were incubated with MitoTracker® Deep Red FM (M22426, Molecular Probes ${ }^{\circledR}$, Life Technologies, Carlsbad, CA, USA) for $45 \mathrm{~min}$ and fixed for 10 min with $4 \%$ paraformaldehyde (15710-S, Electron Microscopy Sciences). Immunofluorescence staining was performed as previously reported [17]. Antibodies are listed in Supplementary Table S2. Images were acquired in a Zeiss LSM510 confocal microscope using the exact same settings for control and experimental samples and analyzed with ImageJ $1.42 \mathrm{q}$ software (NIH, http://rsb.info.nih.gov/ij). Automatic color level correction was used when required to enhance the contrast. Mitochondrial morphology was classified as tubular, mixed, or round (fragmented) according to published criteria [18]. Images were acquired at $\times 63$ magnification, and 1000-5000 cells were counted by two blinded investigators on 16 randomly selected visual fields from at least two independent experiments, using ImageJ. Tyrosine hydroxylase positive neurons were counted over total BIIItubulin positive neurons at day $50-70$ as previously described [17]. Live images were acquired using the Zoe ${ }^{\mathrm{TM}}$ Fluorescent Cell Imager (BioRad, Hercules, CA, USA) at $\times 20$.

\section{PINK1 Over-expression}

Fibroblasts at 70-90\% confluence were electroporated with the Neon ${ }^{\circledR}$ Transfection System (Invitrogen ${ }^{\mathrm{TM}}$, Waltham, MA, USA), using two pulses of $1500 \mathrm{~V}$ for $20 \mathrm{~ms}$, with wild-type PINK1 (pcDNA-DEST47 PINK1 C-green fluorescent protein (GFP)) [19], from MR Cookson, Addgene no. 13316) or a control GFP plasmid, at $0.5 \mu \mathrm{g} / 10^{6}$ cells. Additional controls in each group received only the pulses. Cells were collected for analysis at 24 and 48 h posttransfection given that expression declined rapidly to baseline levels after $72 \mathrm{~h}$ (data not shown). Electroporation in neural cells was done following the same procedure using two pulses of $1000 \mathrm{~V}$. Neurons were collected for analysis at 4 days to minimize the effect of the electroporation on transcriptional changes.

\section{iPSC and Neuronal Differentiation}

Human-iPSC lines from the two Parkinson patients and one carrier and from age-matched control individuals were derived in our laboratory using lentiviral vectors and differentiated as previously described [17] (see Supplementary Fig. S1). The cell lines have been deposited in the Spanish National Cell Bank and are available at http://www.isciii.es/ISCIII/es. 


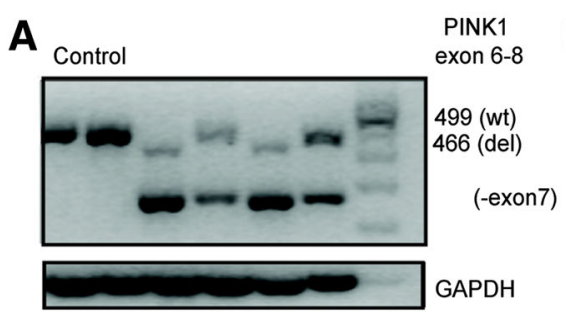

C

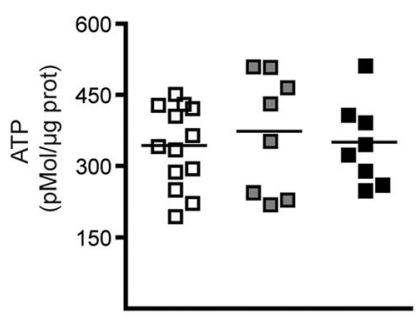

E
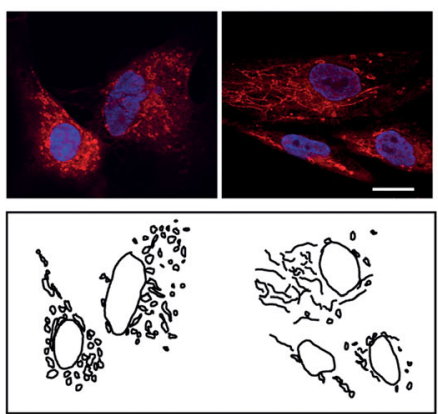

Fig. 1 PINK1 mutant fibroblasts characterization. a PCR analysis and agarose gel electrophoresis in controls, PD patients (PDP1 and PDP3), and carriers (PDP2 and PDP4) show the exon 7 loss and an additional 33bp deletion in PINK1 $1^{\text {-exon7/del }}$ samples (PDP1 and PDP3). GAPDH was used as the reference gene. b Quantitative RT-PCR determination of PINK1 RNA levels in control $(N=4)$, carrier $(N=2)$, and patient $(N=2)$ samples. Columns represent the mean \pm SEM of three independent experiments in the same samples. c ATP levels were not different in control, carrier, and mutant fibroblasts. Scatter plot graphic of three independent experiments. d Glycolysis flux, measured as the rate

\section{Data Analysis and Statistics}

Data analysis was carried out using GraphPad Prism software (v. 4.0c, La Jolla, CA, USA). One-way or two-way ANOVA with Bonferroni post-hoc tests were used to compare groups. Student's $t$ test was used to detect changes in fold expression whenever data were normalized to control levels. In all experiments using human fibroblast samples, four to five control individuals were assayed together with the two $P I N K 1^{\text {-exon7/del }}$ patients and the two $P I N K 1^{\text {-exon7 }}$ carriers or with the two G309D homozygous and one heterozygous carrier in at least two independent determinations. For neuronal experiments, data from three independent differentiations of the three mutant iPSC lines were included with two to three control

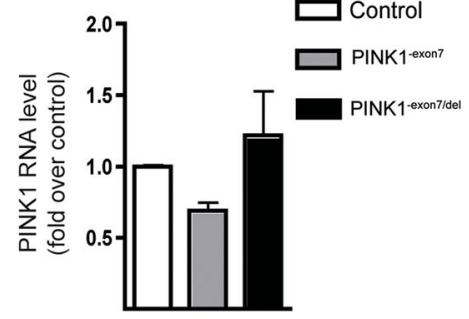

D

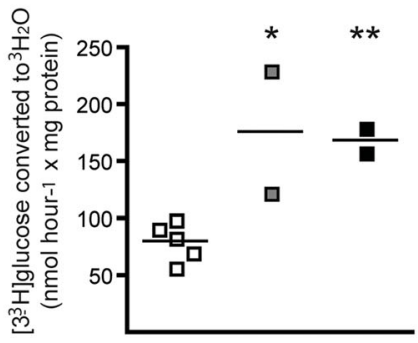

$\mathbf{F}$

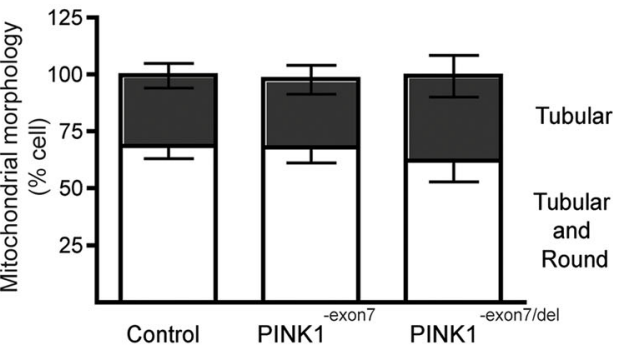

of $\left[3-{ }^{3} \mathrm{H}\right]$ glucose incorporation into ${ }^{3} \mathrm{H}_{2} \mathrm{O}$, in control $(N=5)$, carrier $(N=2)$, and mutant $(N=2)$ fibroblasts, was increased in PINK1 mutant fibroblasts. Experiments were done in triplicate. One-way ANOVA and post-hoc analysis $* p<0.05$ and $* * p<0.01$. e Examples of mitochondrial network tubularity visualized with MitoTracker® staining showing a tubular and round network in the left panel and a tubular in the right one. f. Quantification of tubularity in fibroblast samples showed no differences between genotypes. Mitochondrial morphology was assessed in randomly selected fields, and $>150$ cells were analyzed. Columns represent the mean \pm SEM of three independent experiments. Scale bar $50 \mu \mathrm{m}$

pluripotent lines. In addition to the iPSC lines reprogrammed for this study, control cell lines [17] were differentiated and analyzed in parallel. Data in the figures represent the mean \pm SEM of two to four independent experiments. The threshold for significance was set at $p<0.05$.

\section{Results}

\section{Characterization of Parkinson's Disease PINK1 Mutant Fibroblasts}

We established primary cultures of fibroblasts obtained from dermal biopsies of healthy subjects and individuals carrying 
A

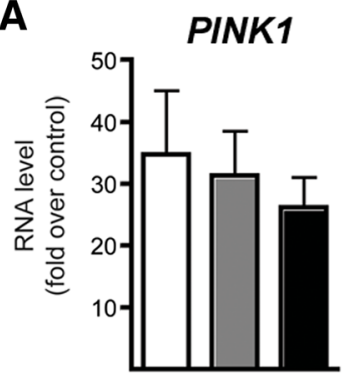

C

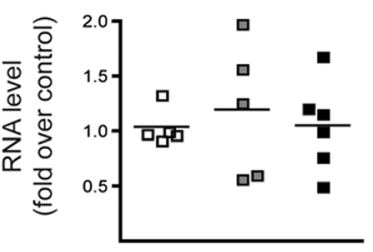

DJ1

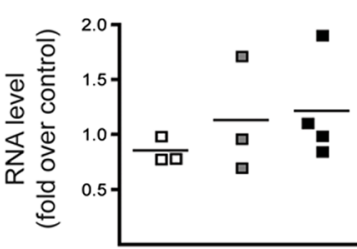

$\square$ Control $\square$ PINK1-exon7 PINK1-exon7del

B

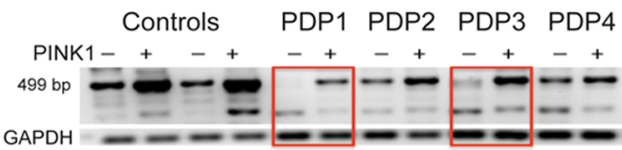

DNM1L

MFF

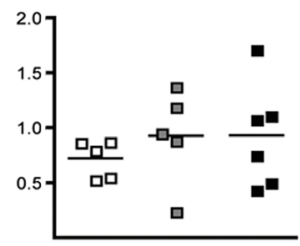

LRRK2

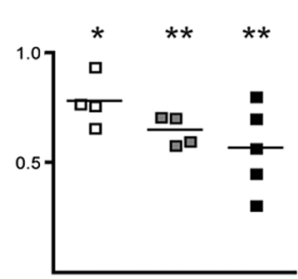

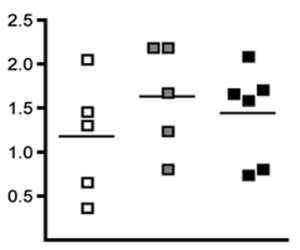

UHRF2

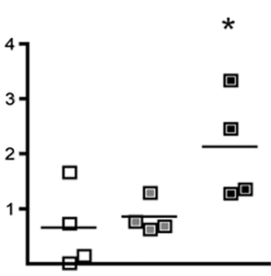

Fig. 2 Over-expression of wild-type PINK1 downregulates $L R R K 2$. a PINK1 RNA levels analyzed by quantitative RT-PCR. Average increase by qPCR was not different across groups $(31 \pm 4.5$-fold increase over mock GFP transfection). b A representative image of an agarose gel electrophoresis showing the expression of the $499 \mathrm{bp}$ band corresponding to the exons 6 to 8 of PINK1 in the PINK1 $1^{- \text {exon7/del }}$ samples (PDP1 and
PDP3; boxed) after electroporation with PINK1.GFP. c RNA levels analyzed by quantitative RT-PCR in fibroblasts after PINK1 over-expression. All data are expressed as fold change over mock (GFP) transfected samples. Scatter plot graphics of two to three independent experiments in control $(N=2)$, carrier $(N=2)$, and mutant $(N=2)$ fibroblasts. One-way ANOVA. $* p<0.05$ and $* * p<0.01$ modifications in the PINK1 gene that result in the inactivation of the normal kinase function [14]. Sample verification was confirmed by conventional PCR analysis (Fig. 1a) that identifies the shorter transcripts corresponding to the deletion (del) and the exon 7 skipping (-exon7) in the compound heterozygous patients (PINK1 $1^{\text {-exon7/del }}$, PDP1 and PDP3) and heterozygous carriers (PINK $1^{\text {-exon7 }}$, PDP2 and PDP4). In spite of the mutations, PINK1 RNA expression was similar in mutant, carriers, and control fibroblasts (Fig. 1b). Baseline ATP levels were not different between groups (Fig. 1c). Nevertheless, using a sensitive method for glycolytic flux assessment, we detected an increase in the glycolytic rate in PINK1 mutants and carriers (Fig. 1d), as recently described in mouse Pink1 KO cells [16]. We next examined the morphology of the mitochondrial network using MitoTracker®; semi-quantitative analysis of tubularity (Fig. 1e, f) showed no differences between genotypes. This does not exclude subtle alterations in mitochondrial morphology or function, but it is in agreement with previous studies in fibroblasts carrying other PINK1 mutations [20]. We analyzed the expression levels of proteins involved in mitochondrial fusion (MNF2) and fission (DNM1L and MFF) to explore whether the lack of morphological changes in mitochondrial tubularity was due to compensatory adjustments in their levels. The results are shown in Supplementary Fig. S1 and revealed minor changes in fusion/ fission dynamics that can reflect compensatory adaptations to the lack of PINK1 kinase function in fibroblasts. These results are consistent with previous studies that have used human fibroblasts to investigate disease and compensatory mechanisms in genetic PD [21].

\section{Effect of PINK1 Over-expression in Fibroblasts}

To identify changes causally related to PINK1 deficiency, we evaluated the capacity of wild-type PINK1 over-expression to modify the gene expression profile in $P I N K 1^{\text {-exon7/del }}$ mutant fibroblasts. Following electroporation, average PINK1 levels determined by qPCR were elevated, $31 \pm 4.5$-fold over control 
A
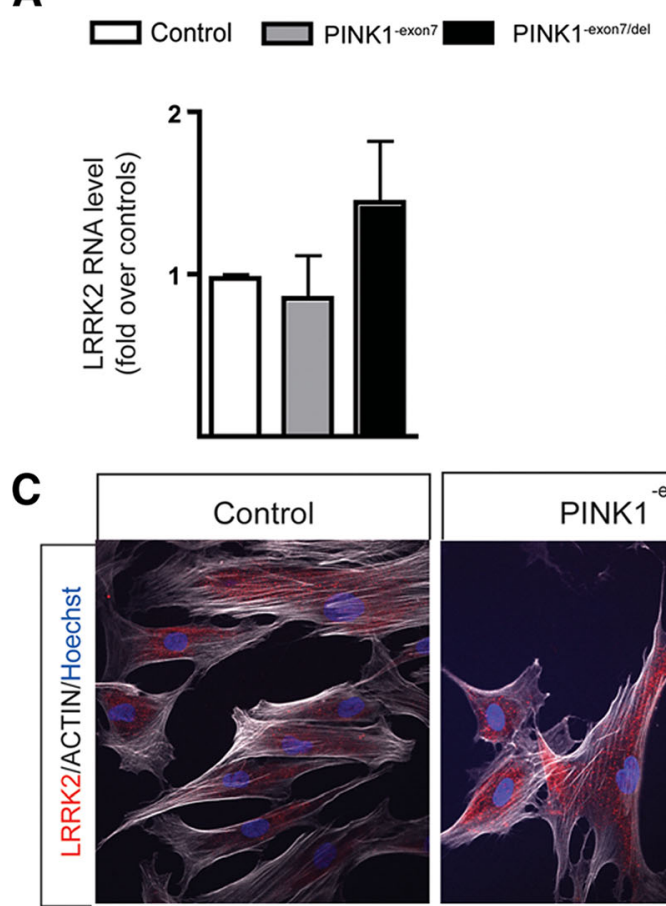

Fig. 3 LRRK2 baseline expression in fibroblasts. a $L R R K 2$ RNA levels by qPCR were not significantly different in control $(N=4)$, carrier $(N=2)$, and mutant $(N=2)$ fibroblasts examined in triplicate. $\mathbf{b}$ Representative blot and quantification of LRRK2 protein level in the same samples showed a significant increase in PINK1 mutants $50 \mu \mathrm{m}$
B
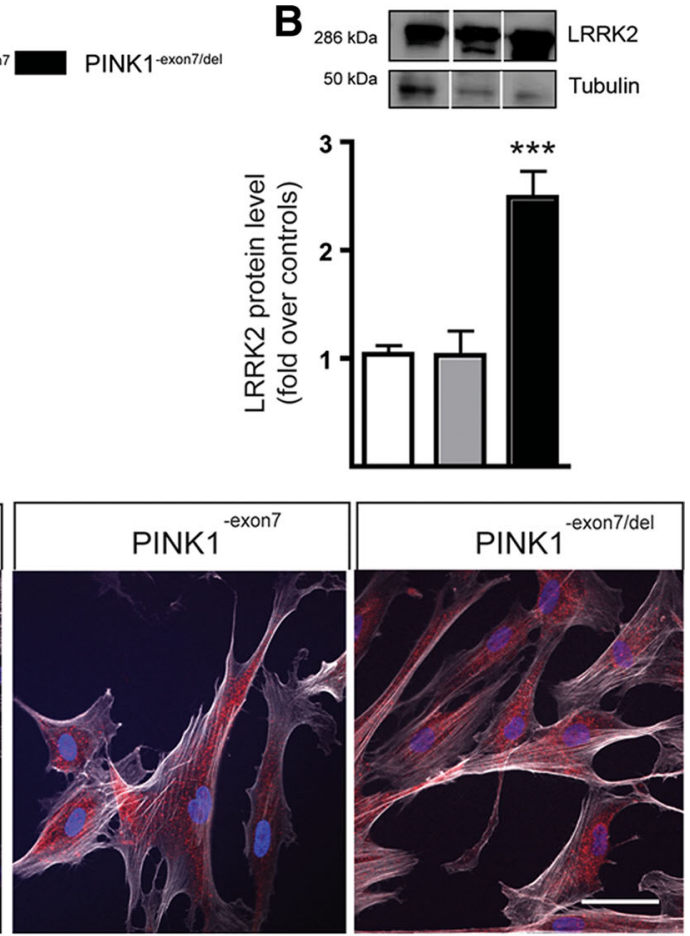

$(2.49 \pm 0.24 ; * * * p<0.001)$. Columns represent the mean \pm SEM of four independent experiments. c LRRK2 subcellular distribution showed a similar pattern in all genotypes. Cells were counterstained with phalloidin (actin, white) to visualize the cytoskeleton. Scale bar cells, with no differences across genotypes in two independent experiments (Fig. 2a) and a clear expression of a 499 band corresponding to the wild-type PINK1 exons 6-8 in transfected mutant fibroblasts (boxed in Fig. 2b). Transient transfection of wild-type PINK1 did not modify the levels of genes involved in mitochondrial dynamics such as mitofusin 2 (MFN2), and the pro-fission DNM1L or MFF genes (Fig. 2c). Likewise, there were no significant changes in DJ1 (PARK7) or Parkin (PARK2, not shown), whereas, interestingly, we found a significant decrease in LRRK2 (PARK8). In view of these results, we analyzed the expression of $U H R F 2$, an E3 ligase reported to be repressed in LRRK2 ${ }^{\mathrm{G} 2019 \mathrm{~S}}$ mutant neurons [22] and found that it was upregulated (Fig. 2d), suggesting that the changes in $L R R K 2$ expression in this paradigm are relevant.

\section{LRRK2 Expression in PINK1 Mutant Fibroblasts}

Since we identified a significant decrease in LRRK2 in PINK1 over-expression experiments, we went on to study the baseline expression of LRRK2 in mutant fibroblasts. LRRK2 RNA was elevated in PINK $1^{\text {-exon7/del }}$ fibroblasts although not significantly different from the control (Fig. 3a). Although some transcripts have been shown to increase with aging in fibroblasts and other tissues, we have not found any correlation between age and LRRK2 mRNA levels in fibroblasts from control individuals studied in our laboratory (age range 1763 years; $R^{2}=0.082, p=0.42$, data not shown). LRRK2 protein was remarkably increased in PINK $1^{\text {-exon7/del }}$ samples, with no change in PINK1 ${ }^{\text {-exon7 }}$ fibroblasts (Fig. 3b). LRRK2 sub-cellular distribution was similar in all groups (Fig. 3c).

\section{LRRK2 Expression in PINK1 Mutant Neurons}

Our findings in fibroblasts suggest that PINK1 and LRRK2 may act in a convergent pathway, with PINK1 regulating LRRK2 expression. Thus, to identify whether LRRK2 may be a factor contributing to the degeneration of dopamine neurons in PINK1-PD, we made iPSC lines from the two PINK1 ${ }^{-}$ exon7/del patients (PDP1 and PDP3 lines) and one carrier (PDP2) (Supplementary Fig. S2 and Fig. 4a, b). Unfortunately, the other carrier line (PDP4) was lost due to technical problems. Pluripotent cells were differentiated towards dopaminergic neurons using an inductive protocol combining developmental signals as described [17] (Fig. 4c). Because significant changes in the expression of genes and levels of proteins occur during neuronal maturation, we analyzed cultures at two time points, corresponding to neural progenitors (2-6 weeks) and neurons (6-12 weeks) (Fig. 4c). There were no apparent defects either in neural 
A

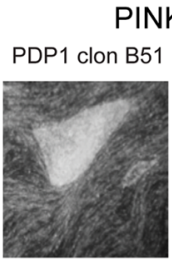

PINK1-exon7/de

PINK1-exon7
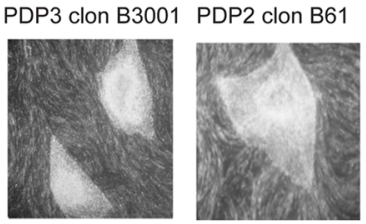

Control IPS

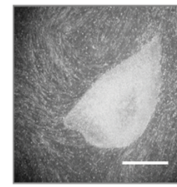

B

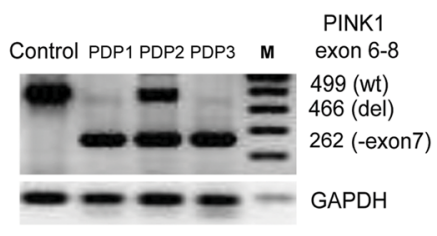

C
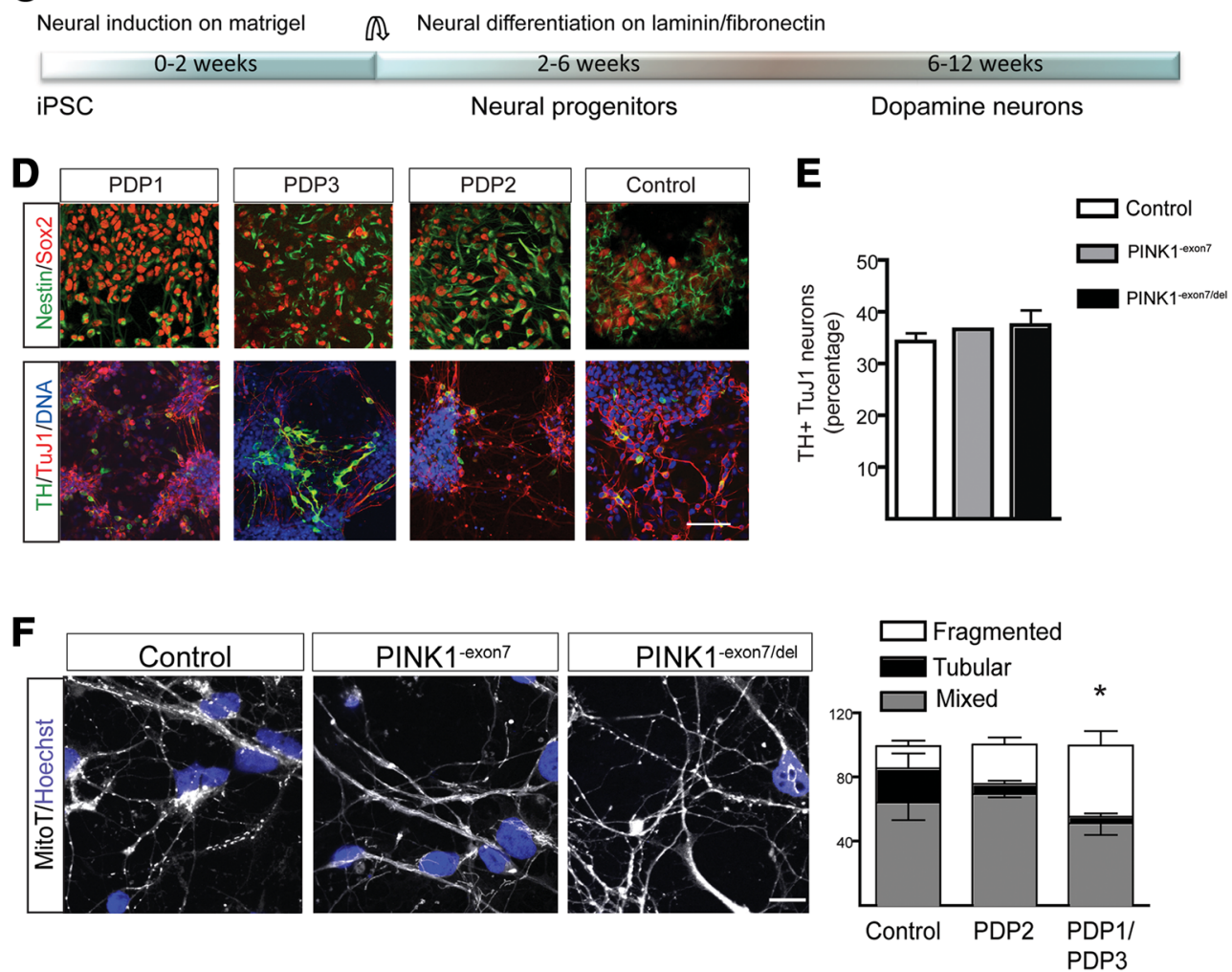

Fig. 4 PINK1 iPSC neuron characterization. a Representative bright field images of iPSC colonies from selected clones from two Parkinson's disease patients (PDP1 and PDP3) and one asymptomatic carrier (PDP2) and a control used for differentiation. Scale bar $0.5 \mathrm{~mm}$. b PCR analysis of PINK1 showed the same splicing pattern in the iPS cell lines as in the original fibroblasts. $\mathbf{c}$ Schematic representation of the differentiation protocol and the two maturation stages used for analysis. $\mathbf{d}$ Confocal images show ubiquitous expression of Sox 2 (red) and Nestin (green) at the neural progenitor stage and $\beta \mathrm{III}-$ tubulin $(\mathrm{TuJ} 1$, red $)$ and $\mathrm{TH}$

induction in the mutant cells, in agreement with published results for iPSC lines carrying homozygous point mutations in the PINK1 gene [7, 23, 24], or in the generation of dopamine neurons, with $>30 \%$ of $\mathrm{TH}$ positive neurons in all genotypes (Fig. 4d, e). Analysis of the mitochondrial morphology in mature neurons showed more cells with a fragmented mitochondrial network in the PINK1 $1^{\text {-exon7/del }}$ cultures (Fig. 4f). However, RNA expression level of $M N F 2, D N M 1 L$, and $M F F$ were not significantly different between genotypes (Supplementary Fig. S3).

It is known that $L R R K 2$ expression is rather low in the developing brain [25]. Indeed, in neural progenitors, $L R R K 2$ RNA levels were barely detectable and there were no (green) at the neuronal stage. Scale bar $50 \mu \mathrm{m}$. e Quantification of TH positive neurons (over TuJ1) at the neuronal stage showed no differences across different cell lines in two independent experiments. $f$ Representative images of MitoTracker ${ }^{\circledR}$ labeling in 8-week-old neurons from control $(N=2)$, carrier $(N=1)$, and mutant $(N=2)$ lines. Scale bar $10 \mu \mathrm{m}$. Quantification of cells presenting predominantly tubular, mixed, or fragmented mitochondrial morphology showed that there were more neurons with fragmented network in the mutant cultures (one-way ANOVA and post-hoc analysis, $p<0.05$ )

differences between groups. The expression increased at the neuronal stage, but only mutant neurons had significantly higher LRRK2 levels than progenitors, which were also higher than control neurons (Fig. 5a). At the protein level, the PINK1 ${ }^{-}$ exon7/del mutant neurons showed increased protein levels of LRRK2 at the neuronal stage (Fig. 5b), corroborating our findings in fibroblasts in a disease-relevant cell context. Over-expression of PINK1 wild-type in $P I N K 1^{\text {-exon7/del }} \mathrm{mu}-$ tant cells was performed at the end of neural progenitor stage (5-6 weeks) with good survival and robust expression of the GFP reporter 1 week after transfection (Fig. 5c) that was confirmed by the presence of a strong band corresponding to the full length PCR product of exons $6-8$ (Fig. 5d). Like in the 
A

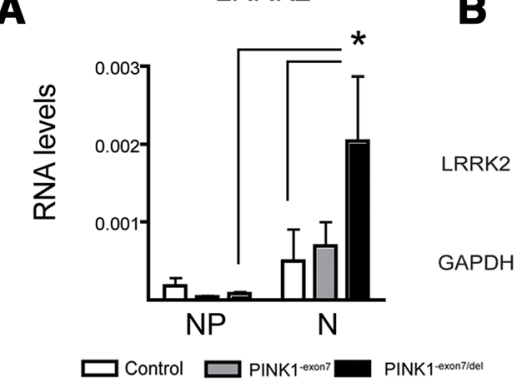

B

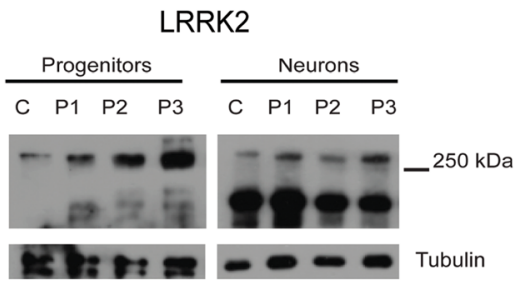

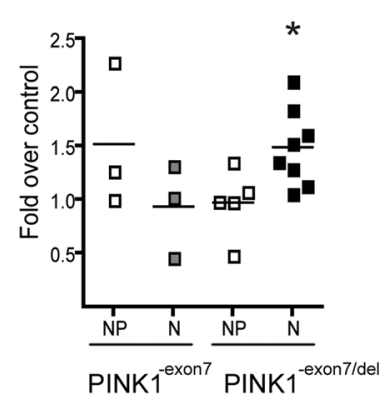

E

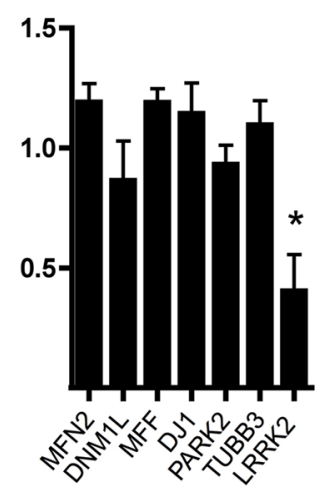

Fig. 5 LRRK2 increase in PINK1 iPSC-derived neurons. a LRRK2 RNA levels significantly increased in PINK1 mutants from the progenitor $(N P)$ to the neuronal $(N)$ stage (two-way ANOVA, $p<0.05$ ) and were higher in mutants than in control neurons $(p<0.05)$ b Representative Western blots and corresponding quantification of LRRK2 protein levels at $N P$ and $N$ stages showed a significant increase in the mutant neurons $(p<0.01)$. $\mathbf{c}$ Live images of GFP immunofluorescence in PINK1 mutant NP (in vitro day 35) 6 days after electroporation with wild-type PINK1.GFP. Scale bar $60 \mu \mathrm{m}$. d Representative image of an agarose gel electrophoresis showing the over-expression of the 499 bp band corresponding to the wild-type PCR product (exons 6-8) of PINK1 in electroporated control and PINK $1^{\text {-exon7/del }}$ neurons (PDP3). Average PINK1 increase by qPCR was not different across groups. e qPCR analysis in three independent over-expression experiments showed a significant downregulation of $L R R K 2$ (a decrease of $60 \pm 9.6 \%$ over GFP mock transfection; $p<0.05)$, without inducing any significant changes in fusion/fission genes, PARK7 and PARK2, or $\beta$ III-tubulin (TUBB3) expression

mutations. We did not find consistent mitochondrial alterations as hypothesized but found instead a remarkable deregulation of LRRK2, revealing a previously under-recognized connection between these two PARK genes in human cells. We corroborated this association in fibroblasts from a second family with a different mutation in PINK1.

Our data suggest that PINK1 exerts, directly or indirectly, an inhibitory effect on $L R R K 2$ at the transcriptional level. Indeed, over-expression of wild-type PINK1 decreased $L R R K 2$ levels in control and mutant cells. In mutant fibroblasts and neurons, $L R R K 2$ deregulation resulted in increased LRRK2 protein level.

Abnormal protein synthesis, cytoskeletal dynamics, and mitochondrial transport have all been implicated in LRRK2 pathogenesis [26]. Studies in C. elegans [12] and Drosophila [27] suggest an antagonistic effect of lrk-1 and pink-1 [13] with clear, if complex, interactions between these two genes, not unlike in our study.

In agreement with previous studies [8, 20, 28], PINK1patient fibroblasts showed only subtle signs of mitochondrial dysfunction with normal levels of ATP - at the expense of an increased glycolytic rate - and no conspicuous alterations in
In this study, we sought to define a disease-related in vitro phenotype in human cells with PD-associated PINK1 


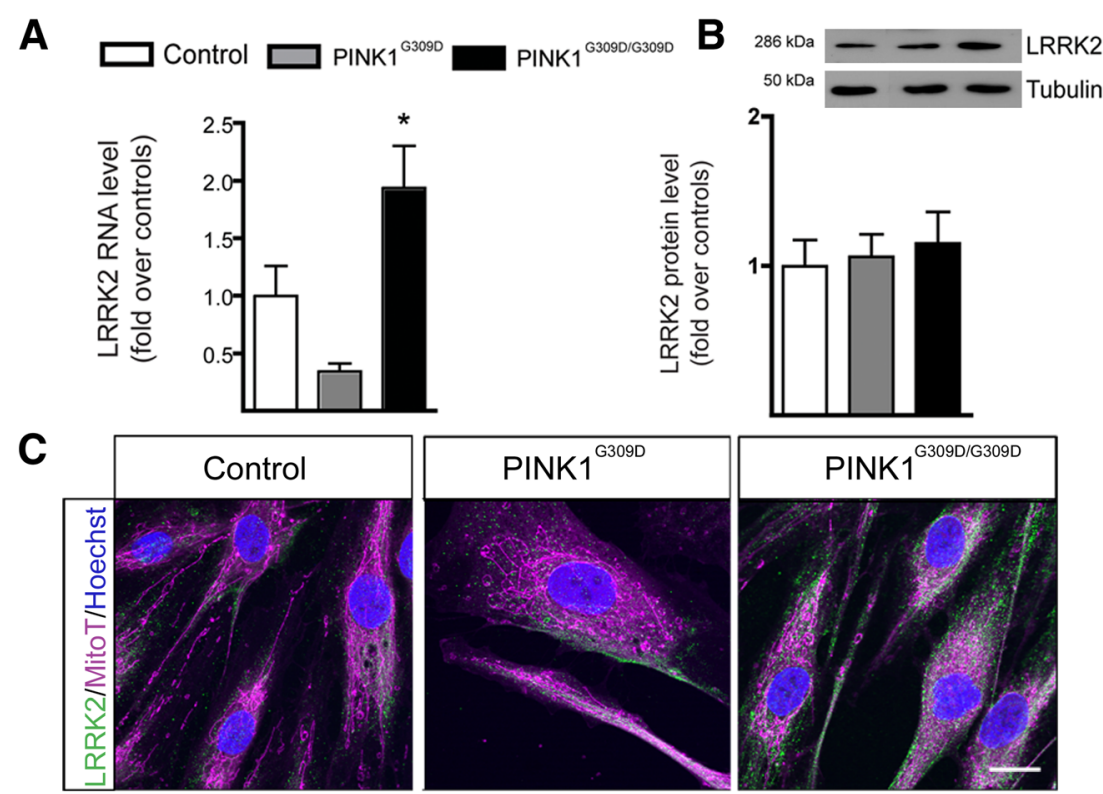

Fig. 6 LRRK2 baseline expression in PINK1 G309D fibroblasts. a $L R R K 2$ RNA levels analyzed by quantitative RT-PCR in fibroblasts with PINK1 G309D homozygous mutations $(N=2)$ were significantly higher than in controls $(N=5)$. Columns represent the mean \pm SEM of three independent experiments. $* p<0.05$. b LRRK2 protein levels in the same samples were not significantly different from control in this analyses; experiments were done in triplicate and a representative blot is shown above the quantification over the loading control. c Representative images of LRRK2 (green) and the mitochondrial network labeled with MitoTracker® (magenta) in the G309D fibroblasts. Scale bar $10 \mu \mathrm{m}$ the mitochondrial network. Similar findings have been documented for other PARK genes, like Parkin [29] and LRRK2 [30]. The net effect of PINK1 kinase deficiency on fusion/ fission dynamics is a matter of controversy as different model organisms show opposite effects (increased tubularity or increased fission) [10]. It is worth noting that there are remarkable differences between vertebrate (mouse and zebrafish) and invertebrate (fly and worm) models regarding the effects of PARK genes on mitochondrial dynamics. Analysis of proteins involved in fusion and fission mitochondrial dynamics in PD fibroblasts showed alterations that are consistent with activation of compensatory mechanisms in the mutants but could also reflect a defective inhibition of fission through TRAP1 [31, 32].

Recent studies have proposed patient fibroblasts as an adequate system to investigate disease mechanisms and compensatory pathways in genetic PD [21], but we took advantage of reprogramming technology to generate iPSC and neurons from these patients and validate our findings in a diseaserelevant cell type. iPSC-derived neurons offer the benefit of preserving cell-type specific endogenous expression and transcriptional regulation of the mutated gene. While modeling a complex, age-related and mostly sporadic disorder such as PD is challenging, iPSC harboring monogenic, early-onset variants like PINK1 may provide valuable insights into disease mechanisms [33]. Also critical is the capacity to generate a significant proportion of target cells from the iPSC. In this study, we obtained about $30 \%$ of $\mathrm{TH}+$ neurons from all genotypes.
PINK $1^{- \text {exon7/del }}$ mutant neurons had a significant increase in the percentage of cells with fragmented mitochondria suggesting a shift in mitochondrial dynamics towards fission. However, this should be interpreted with caution, because in neurons, many different pathways can result in this phenotype. Indeed, we did not find any significant changes in fusion or fission genes, besides a uniform developmental increase in their expression.

Interestingly enough, we observed a remarkable upregulation of LRRK2 in the PINK1 $1^{\text {-exon7/del }}$ mutant neurons. Furthermore, like in fibroblasts, transient over-expression of wild-type PINK1 effectively downregulated $L R R K 2$ expression without having any effect on neuronal markers, mitochondrial dynamics, or other PARK genes.

LRRK2 has been implicated in a very broad range of cellular pathways, and the precise mechanisms leading to neuronal degeneration in PD-associated LRRK2 mutations remain to be defined. Nonetheless, increased levels of LRRK2 appear to be directly related to the pathogenicity/ toxicity of PD-related mutations, at least for the LRRK2 $2^{\mathrm{G} 2019 \mathrm{~S}}$ mutation [34], which is one of the mutations most frequently associated with PD. Importantly, not only mutations but also several polymorphisms in LRRK2 are associated to an increased risk of PD. Our data suggest that PINK1 and LRRK2 act on a common pathogenic pathway in an antagonistic manner.

Both LRRK2 and PINK1 have been found to modulate the phosphorylation state of several Rab GTP-ases [35, 36], and PD-related mutations could therefore affect vesicle trafficking. 
In this regard, it is also interesting that over-expression of Rab1 could rescue the SNCA mutant phenotype in invertebrate and mammalian models and in human neurons carrying PD-associated SNCA triplication [37, 38], indicating that several PARK genes with quite distinct clinical manifestations (early onset, typical PD, or dementia with Lewy body) may share common molecular mechanisms [39].

Further downstream, LRRK2 has recently been found to directly phosphorylate p53 (TP53), thus acting in a proapoptotic role - phosphorylation of $\mathrm{p} 53$ leads to transcriptional activation of pro-apoptotic genes such as $B A X, P U M A$ (BBC3), NOXA (PMAIP1), and others, as well as activation of transcriptional independent pro-apoptotic mechanisms - in a tissue-specific manner [40]. It has been proposed that either LRRK2 or p53 could initiate cell death in dopamine neurons [41]. PINK1 negatively regulates p 53 activity through activation (phosphorylation) of histone deacetylases, which could account for its pro-survival and anti-apoptotic role [42]. Therefore, in PINK1 mutant neurons, the lack of functional PINK1 could perhaps lead to activation of pro-apoptotic mechanisms through upregulation of LRRK2, although this remains to be proven. A better understanding of LRRK2 function is required to identify novel ways to re-establish this balance in susceptible neurons in PINK1-associated and, perhaps, more broadly in PD.

In conclusion, we report a novel role of PINK1 modulating the levels of LRRK2 in patient fibroblasts and neurons. Although LRRK2 is one of the genes that is most frequently associated with PD, its role in early-onset recessive forms of the disease had not been previously determined. Our results suggest a convergent pathway for these PARK genes, acting in an antagonistic manner, and broaden the involvement of LRRK2 in the pathogenesis of PD.

Acknowledgements We are grateful to the patients, relatives, and healthy individuals that participated in the study and to Dr. Angel García Martin for control samples and technical advice.

Authors' Contributions GAz: Acquisition, analysis, and interpretation of data and drafting and revision of manuscript. RLM: Acquisition and analysis of data and drafting and revision of manuscript. PR: Acquisition and analysis of data and drafting and revision of manuscript. AS: Acquisition and analysis of data. NV: Acquisition and analysis of data. AZ: Acquisition and analysis of data. DJB: Acquisition and analysis of data. JPB: Supervision and data analysis, interpretation, and critical revision of manuscript. BM: Critical revision of manuscript. GAu: Data interpretation and critical revision of manuscript. JMA: Study conception and critical revision of manuscript. RSP: Study design, data acquisition, analysis and interpretation, and drafting and critical revision of manuscript. All: Revision and approval of manuscript.

Compliance with Ethical Standards The study was approved by the Ethical Committee on the Use of Human Subjects in Research in Euskadi, Spain. All subjects gave informed consent for the study using forms approved by the ethical committees on the Use of Human Subjects in Research at Hospital Universitario Insular de Gran Canaria, La Palmas de Gran Canaria; Hospital Donostia and Onkologikoa, San Sebastián; and Hospital San Cecilio, Granada, respectively.
Conflict of Interest The authors declare that they have no competing interests.

Funding This study is funded by grants from the European Commission, CORDIS FP7, HEALTH-2, 278871 (DDPDGENES), and the Joint Program in Neurodegenerative Diseases (DAMNDPATHS) to RSP. JPB is the recipient of grants from MINECO (SAF2013-41177-R) and the NIH/NIDA (1R21DA037678-01). GAu is the recipient of grants from DFG (AU96/10-1) and the German National Genome Research Network NGFNplus (Neuron-Parkinson-subproject 7).

Open Access This article is distributed under the terms of the Creative Commons Attribution 4.0 International License (http:// creativecommons.org/licenses/by/4.0/), which permits unrestricted use, distribution, and reproduction in any medium, provided you give appropriate credit to the original author(s) and the source, provide a link to the Creative Commons license, and indicate if changes were made.

\section{References}

1. Farrer MJ (2006) Genetics of Parkinson disease: paradigm shifts and future prospects. Nat Rev 7:306-318

2. Berg D, Lang AE, Postuma RB et al (2013) Changing the research criteria for the diagnosis of Parkinson's disease: obstacles and opportunities. Lancet Neurol 12:514-524

3. Valente EM, Abou-Sleiman PM, Caputo V et al (2004) Hereditary early-onset Parkinson's disease caused by mutations in PINK1. Science 304:1158-1160

4. Pilsl A, Winklhofer KF (2012) Parkin, PINK1 and mitochondrial integrity: emerging concepts of mitochondrial dysfunction in Parkinson's disease. Acta Neuropathol 123:173-188

5. Haque ME, Thomas KJ, D’Souza C et al (2008) Cytoplasmic Pink1 activity protects neurons from dopaminergic neurotoxin MPTP. Proc Natl Acad Sci U S A 105:1716-1721

6. Yu W, Sun Y, Guo S et al (2011) The PINK1/Parkin pathway regulates mitochondrial dynamics and function in mammalian hippocampal and dopaminergic neurons. Hum Mol Genet 20:32273240

7. Rakovic A, Shurkewitsch K, Seibler P et al (2013) Phosphatase and tensin homolog (PTEN)-induced putative kinase 1 (PINK1)-dependent ubiquitination of endogenous Parkin attenuates mitophagy: study in human primary fibroblasts and induced pluripotent stem cell-derived neurons. J Biol Chem 288:2223-2237

8. Morais VA, Haddad D, Craessaerts K et al (2014) PINK1 loss-offunction mutations affect mitochondrial complex I activity via NdufA10 ubiquinone uncoupling. Science 344:203-207

9. Van Laar VS, Berman SB (2013) The interplay of neuronal mitochondrial dynamics and bioenergetics: implications for Parkinson's disease. Neurobiol Dis 51:43-55

10. Scarffe LA, Stevens DA, Dawson VL et al (2014) Parkin and PINK1: much more than mitophagy. Trends Neurosci 37:315-324

11. Paisan-Ruiz C, Lewis PA, Singleton AB (2013) LRRK2: cause, risk, and mechanism. J Parkinsons Dis 3:85-103

12. Saha S, Guillily MD, Ferree A et al (2009) LRRK2 modulates vulnerability to mitochondrial dysfunction in Caenorhabditis elegans. J Neurosci 29:9210-9218

13. Samann J, Hegermann J, von Gromoff E et al (2009) Caenorhabditits elegans LRK-1 and PINK-1 act antagonistically in stress response and neurite outgrowth. J Biol Chem 284:1648216491 
14. Samaranch L, Lorenzo-Betancor O, Arbelo JM et al (2010) PINK1linked parkinsonism is associated with Lewy body pathology. Brain 133:1128-1142

15. Lopez de Maturana R, Aguila JC, Sousa A et al (2014) Leucine-rich repeat kinase 2 modulates cyclooxygenase 2 and the inflammatory response in idiopathic and genetic Parkinson's disease. Neurobiol Aging 35:1116-1124

16. Requejo-Aguilar R, Lopez-Fabuel I, Fernandez E et al (2014) PINK1 deficiency sustains cell proliferation by reprogramming glucose metabolism through HIF1. Nat Commun 5:4514

17. Aguila JC, Blak A, van Arensbergen J et al (2014) Selection based on FOXA2 expression is not sufficient to enrich for dopamine neurons from human pluripotent stem cells. Stem Cells Transl Med 3: $1032-1042$

18. Zhao J, Liu T, Jin S et al (2011) Human MIEF1 recruits Drp1 to mitochondrial outer membranes and promotes mitochondrial fusion rather than fission. EMBO J 30:2762-2778

19. Beilina A, Van Der Brug M, Ahmad R et al (2005) Mutations in PTEN-induced putative kinase 1 associated with recessive parkinsonism have differential effects on protein stability. Proc Natl Acad Sci U S A 102:5703-5708

20. Hoepken HH, Gispert S, Morales B et al (2007) Mitochondrial dysfunction, peroxidation damage and changes in glutathione metabolism in PARK6. Neurobiol Dis 25:401-411

21. Auburger G, Klinkenberg M, Drost J et al (2012) Primary skin fibroblasts as a model of Parkinson's disease. Mol Neurobiol 46: 20-27

22. Reinhardt P, Schmid B, Burbulla LF et al (2013) Genetic correction of a LRRK2 mutation in human iPSCs links parkinsonian neurodegeneration to ERK-dependent changes in gene expression. Cell Stem Cell 12:354-367

23. Seibler P, Graziotto J, Jeong H et al (2011) Mitochondrial Parkin recruitment is impaired in neurons derived from mutant PINK1 induced pluripotent stem cells. J Neurosci 31:5970-5976

24. Miller JD, Ganat YM, Kishinevsky S et al (2013) Human iPSCbased modeling of late-onset disease via progerin-induced aging. Cell Stem Cell 13:691-705

25. Giesert F, Hofmann A, Burger A et al (2013) Expression analysis of Lrrk1, Lrrk2 and Lrrk2 splice variants in mice. PLoS One 8:e63778

26. Esteves AR, Swerdlow RH, Cardoso SM (2014) LRRK2, a puzzling protein: insights into Parkinson's disease pathogenesis. Exp Neurol 261:206-216

27. Venderova K, Kabbach G, Abdel-Messih E et al (2009) Leucinerich repeat kinase 2 interacts with Parkin, DJ-1 and PINK-1 in a Drosophila melanogaster model of Parkinson's disease. Hum Mol Genet 18:4390-4404
28. Exner N, Treske B, Paquet D et al (2007) Loss-of-function of human PINK1 results in mitochondrial pathology and can be rescued by parkin. J Neurosci 27:12413-12418

29. Zanellati MC, Monti V, Barzaghi C et al (2015) Mitochondrial dysfunction in Parkinson disease: evidence in mutant PARK2 fibroblasts. Front Genet 6:78

30. Mortiboys H, Furmston R, Bronstad G et al (2015) UDCA exerts beneficial effect on mitochondrial dysfunction in LRRK2G2019S carriers and in vivo. Neurology 85:846-852

31. Pridgeon JW, Olzmann JA, Chin LS et al (2007) PINK1 protects against oxidative stress by phosphorylating mitochondrial chaperone TRAP1. PLoS Biol 5:e172

32. Takamura H, Koyama Y, Matsuzaki S et al (2012) TRAP1 controls mitochondrial fusion/fission balance through Drp1 and Mff expression. PLoS One 7:e51912

33. Badger JL, Cordero-Llana O, Hartfield EM et al (2014) Parkinson's disease in a dish-using stem cells as a molecular tool. Neuropharmacology 76 Pt A:88-96

34. Skibinski G, Nakamura K, Cookson MR et al (2014) Mutant LRRK2 toxicity in neurons depends on LRRK2 levels and synuclein but not kinase activity or inclusion bodies. J Neurosci 34:418 433

35. Lai YC, Kondapalli C, Lehneck R et al (2015) Phosphoproteomic screening identifies Rab GTPases as novel downstream targets of PINK1. EMBO J 34:2840-2861

36. Steger M, Tonelli F, Ito $\mathrm{G}$ et al (2016) Phosphoproteomics reveals that Parkinson's disease kinase LRRK2 regulates a subset of Rab GTPases. eLife 5:e12813

37. Cooper AA, Gitler AD, Cashikar A et al (2006) Alpha-synuclein blocks ER-Golgi traffic and Rab1 rescues neuron loss in Parkinson's models. Science 313:324-328

38. Mazzulli JR, Zunke F, Isacson O et al (2016) Alpha-synucleininduced lysosomal dysfunction occurs through disruptions in protein trafficking in human midbrain synucleinopathy models. Proc Natl Acad Sci U S A 113:1931-1936

39. Clague MJ, Rochin L (2016) Parkinson's disease: a traffic jam? Curr Biol 26:R332-R334

40. Ho DH, Kim H, Kim J et al (2015) Leucine-rich repeat kinase 2 (LRRK2) phosphorylates p53 and induces p21(WAF1/CIP1) expression. Mol Brain 8:54

41. Levine AJ, Harris CR, Puzio-Kuter AM (2012) The interfaces between signal transduction pathways: IGF-1/mTor, p53 and the Parkinson disease pathway. Oncotarget 3:1301-1307

42. Choi HK, Choi Y, Kang H et al (2014) PINK1 positively regulates HDAC3 to suppress dopaminergic neuronal cell death. Hum Mol Genet 24:1127-1141 